\title{
Primary Small Bowel Melanoma. Literature Review and a Case Report
}

\author{
Alma Llukacaj ${ }^{1}$, Henri Kolani ${ }^{2}$, Etmont Celiku $^{3}$, Eljona Xhelili ${ }^{4}$ \\ ${ }^{1}$ First Service of General Surgery, Department of General and Digestive Surgery, Master Degree in Surgery, \\ Hospital University Center "Mother Theresa", Tirana, Albania \\ ${ }^{2}$ First Service of General Surgery, Department of General and Digestive Surgery, PhD in Surgery \\ Hospital University Center "Mother Theresa", Tirana, Albania \\ ${ }^{3}$ Head of the First Service of General Surgery, Department of General and Digestive Surgery, \\ Professor of Surgery, Hospital University Center "Mother Theresa”, Tirana, Albania \\ ${ }^{4}$ First Service of General Surgery, Department of General and Digestive Surgery, \\ Resident of Surgery, Hospital University Center "Mother Theresa", Tirana, Albania
}

\begin{abstract}
Small bowel melanoma (SBM) is a rare entity, most of the time poorly diagnosed, and therefore it presents itself late in the course of the disease. Whether arise primarily or metastatically from a cutaneous melanoma, it is difficult to establish. In this report, we present a rare case of primary SBM and review of the current literature. A 50-year-old man is presented with melena and severe anemia in the emergency department. A series of investigations including abdominal ultrasonography (US), esophageal-gastroduodenoscopy (EGDS) and colonoscopy were normal. Abdominal computed tomography showed a prominent mass which urged for immediate surgery. On laparotomy, the mass was resected, and histologically diagnosed as melanoma. Meticulous postoperative clinical examination revealed no cutaneous lesions. Review of the literature discusses gastrointestinal malignant melanoma and the possibilities of existence of true primary intestinal melanoma.
\end{abstract}

Keywords: small bowel, gastrointestinal melanoma, surgery, primary tumor, melanocyte

\section{Introduction}

Most melanomas of the small intestine are metastases from primary cutaneous lesions, but melanoma can also develop as a primary mucosal neoplasm in the gastrointestinal tract. Therefore malignant melanoma originating primary from the small intestine is extremely rare. Malignant melanomas are relatively common cancers making up around $2 \%$ of all tumors [1]. The vast majority of melanomas are cutaneous but non-cutaneous tumors such as ocular, leptomeningeal, oral, nasopharyngeal, esophageal, bronchial, vaginal, anorectal and nail-bed melanomas (in descending order of frequency) occur, very rarely [2]. Only $3-4 \%$ of all melanomas originate in mucosal membranes as primaries [3]. Symptoms are generally identical to those caused by other GI tumors, including abdominal pain, fatigue, dysphagia, constipation, tenesmus, small bowel obstruction, perforated bowel, hematemesis, and melena. We report a case of malignant melanoma primary presented to the GI tract, followed by a review of the literature.

\section{Case Report}

A 50 years old man is presented to the emergency department with fatigue, weakness, dull abdominal pain in the lower quadrants and left flank, nausea and one episode of lipothymia after an abundant liquid melanoic discharge. He reported weight loss and anorexia in the last two months. His medical history was unremarkable, never been hospitalized before. Primary examination revealed skin paleness and tachycardia, while the clinical examination of the abdomen showed normal intestinal sounds and a painful abdomen on the lower quadrants. Rectal examination was positive for melena. The basic biochemical profiles were within the framework of normal. The hematological profile relieved a severe anemia. The basic values were $\mathrm{RBC}=2.47 \times 10^{6} / \mathrm{mm}^{3}$, $\mathrm{HGB}=5.6 \mathrm{~g} / \mathrm{dl}, \quad \mathrm{HCT}=19.1 \%, \quad \mathrm{PLT}=549 \times 10^{3} / \mathrm{mm}^{3}, \quad$ seric ferritin $=16,02 \mathrm{ng} / \mathrm{ml}$. An abdominal ultrasound performed in the emergency unit showed a huge mass which extended from the left iliac fossa to the left flank. The patient was hospitalized and more specific examination were submitted, which included a colonoscopy and a esophageal gastro duodenoscopy (EGDS), both with normal findings.

A computed tomography $\mathrm{CT}$ with intravenous contrast showed a mass of the small bowels, raised from the left iliac fossa to the left flank which measured $14 \times 9 \mathrm{~cm}$, with inflammatory image of an abscess. The examination found enlarged lymph nodes in the surrounding area and also lowmoderate quantity of free liquid in the pelvic area. Other abdominal organs were normal. Tumor marker assays showed a $\mathrm{CEA}=1,07 \mathrm{ng} / \mathrm{ml}$ and a CA19-9=5,07U/ml, which fell within normal limits. The inflammatory markers such as VES and PCR were normal. The patient went therefore to surgery.

The intraoperative finding (Images 1,2) was a mass of $14 \times 10 \mathrm{~cm}$ which extended from the jejunum, that occupied almost $2.5 \mathrm{~m}$ of the small bowel, malodorous, friable, colored bluish to wine as showed in the images below. 


\section{International Journal of Science and Research (IJSR) \\ ISSN (Online): 2319-7064}

Index Copernicus Value (2013): 6.14 | Impact Factor (2015): 6.391

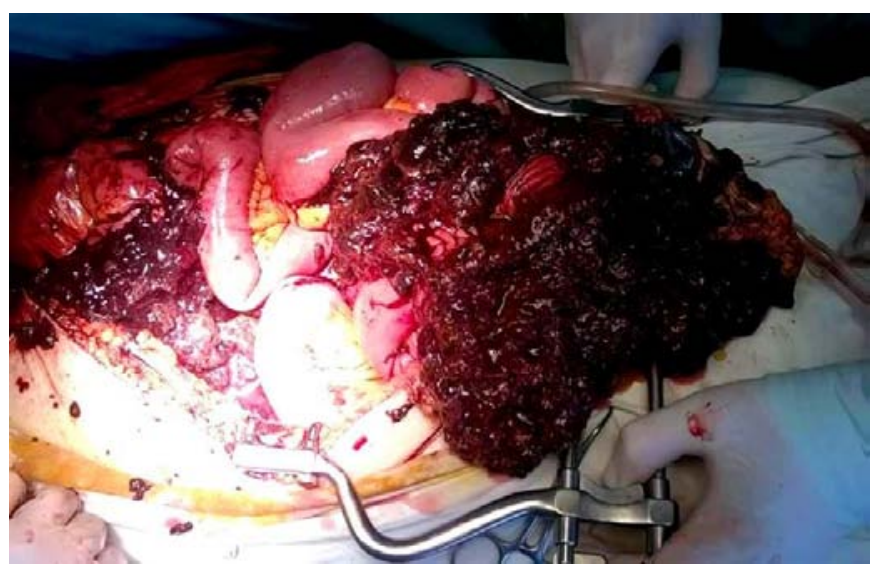

Image 1

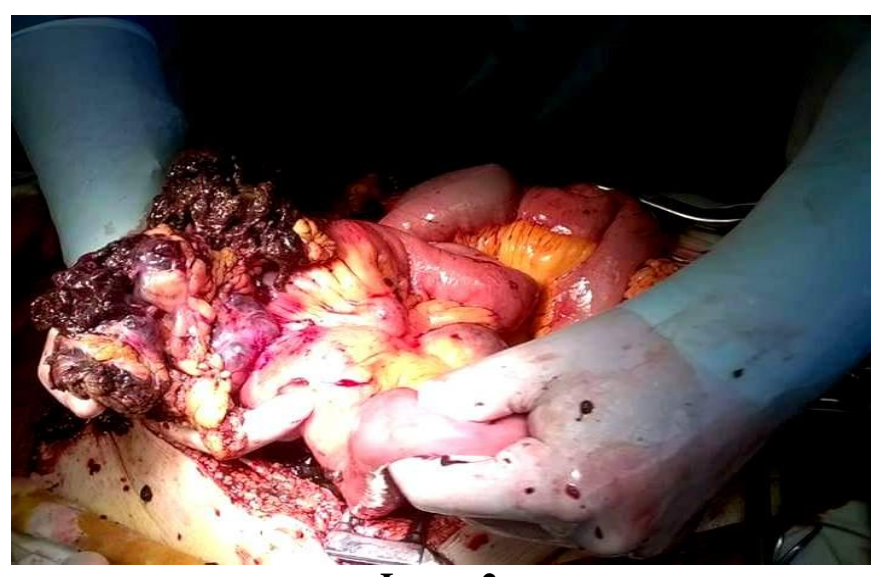

Image 2

Images taken during the surgery show a tumor with friable tissue, hemorrhagic, ulcerative. Small bowel resection with macroscopically free disease margins was performed, followed by a side-to-side jejuno-ileal anastomosis. Panels of immunohistochemical markers showed positive in HMB45 $(+)$ Melan-A (+) and S100 (+). The histological report highlighted small intestine malignant melanoma (Images 3, 4, 5). The examination of skin, eyes, esophagus and anus was negative for primary melanoma and the investigation of the chest, abdomen and brain with a full body CT-scan as well as the bone schintigraphy did not report metastatic disease. The patient's medical history did not include excision of any skin lesions. Therefore the final diagnosis was primary melanoma of the small intestine.

Four months later, patient died from the metastatic disease.

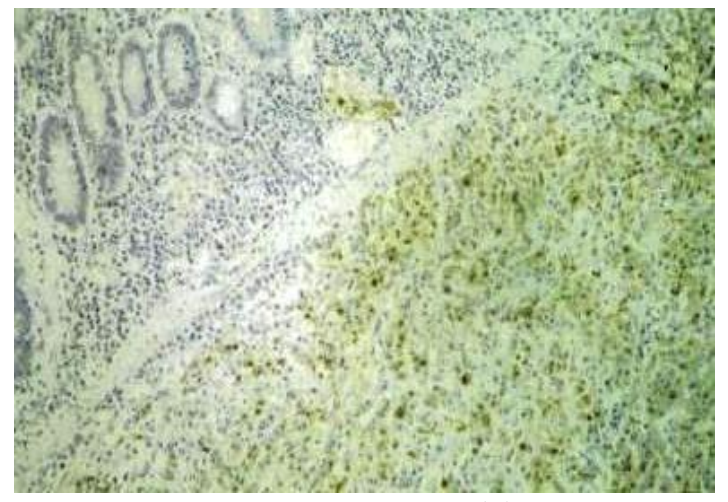

Image 3: HMB-45(+)

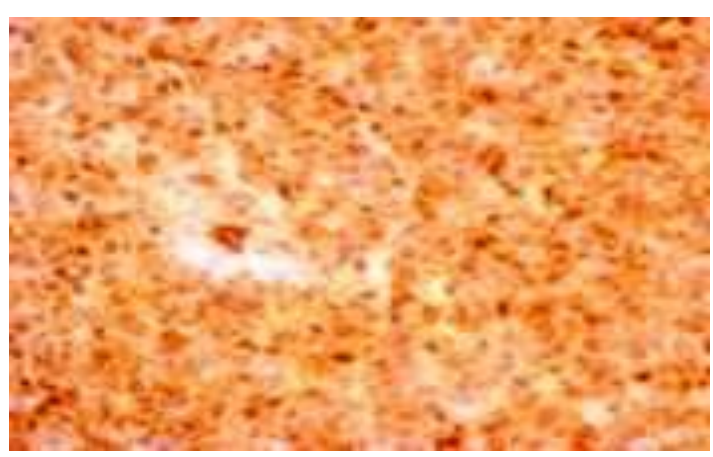

Image 4: S100 (+)

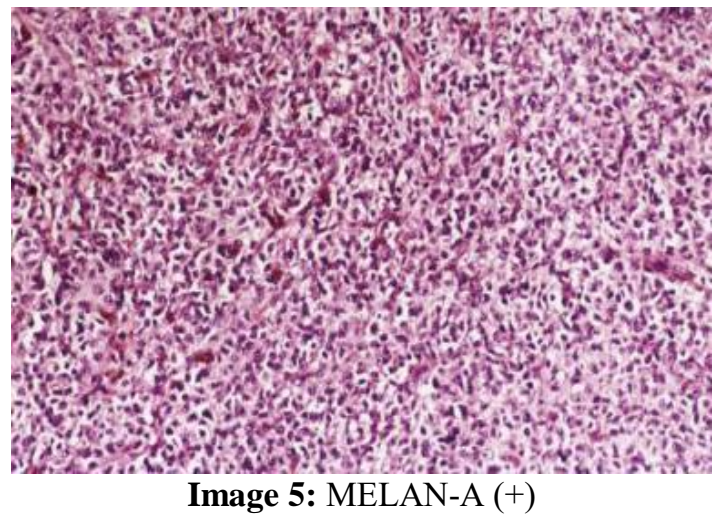

\section{Discussions}

Malignant melanoma that involves the GI tract may be either primary or metastatic [4]. Primary GI melanoma can arise in various GI mucosal sites, including the oral cavity, esophagus, small bowel, colon, rectum and anus, in the absence of prior cutaneous melanoma [5]. These regions have been documented by immune histochemical stains such as HMB-45 and S100 that contain melanocytes. Primary melanomas of the GI tract are rarely diagnosed at an early stage, tend to be more aggressive, and are associated with a worse prognosis. Distinguishing between a primary GI mucosal melanoma and a melanoma metastatic to the GI tract from an unknown or regressed cutaneous primary may be difficult [4]. The diagnosis of primary mucosal melanoma is pathologically supported if a precursor lesion or melanosis is present, such as junctional melanocytic proliferation within the mucosa. In contrast, metastasis from spontaneous regression of primary cutaneous melanoma is pathologically characterized by lymphocytic infiltration of the dermis and the presence of melanophages, vascular proliferation, and reparative fibrosis [3]. Clinically, a primary GI mucosal melanoma is suggested if the patient has no obvious primary cutaneous melanoma or has an isolated GI lesion without other metastases out of the GI tract.

Gastrointestinal melanomas are usually asymptomatic in their early stages. Diagnosis is frequently delayed until the occurrence of a potentially life-threatening complication, such as intestinal intussusception, obstruction, bleeding or perforation [15]. For most patients with metastatic GIT melanoma, a surgical procedure is performed on an emergency basis [16].

In our case, the patient didn't present typical symptoms

\section{Volume 5 Issue 7, July 2016 www.ijsr.net}




\section{International Journal of Science and Research (IJSR) \\ ISSN (Online): 2319-7064}

Index Copernicus Value (2013): 6.14 | Impact Factor (2015): 6.391

linked to intestinal obstruction. CT scan revealed lower intestinal mass with primary hypothesis of an abscess, but no extensive intestinal dilatation.

In contrast to primary gastrointestinal melanoma, which is characteristically solitary, gastrointestinal metastases from cutaneous melanoma are often multiple and co-existent with metastases to additional systems. Secondary small intestinal melanoma can be infiltrating, polypoid, cavitating or exoenteric and any type can be either pigmented or nonpigmented [20].

The common feature of all melanomas is the cell of origin, the melanocyte. Melanocytes are pigmented dendritic-like cells located in several anatomic sites. These sites include the epidermis, the eye, and epithelia of the nasal cavity, oropharynx, anus, vagina and urinary tract. In addition to these sites, melanocytes have been demonstrated in Meckel's diverticulum by immunoperoxidase studies [19]. Normally, the small bowel and colon contain no melanocytes. Melanomas of the small bowel are thought to originate from melanoblastic cells of the neural crest, which migrate to the distal ileum through the umbilical-mesenteric canal. In the gut, neural crest cells differentiate into amine precursor uptake and decarboxylation (APUD) cells, which can undergo neoplastic transformation and produce tumors such as carcinoids or gastrinomas. Melanomas may potentially originate from APUD cells, although they do not, unlike the usual APUDomas, produce any hormones. According to the APUD theory, the ileum, which represents the distal end of the umbilicalmesenteric canal, should be the most common site of primary malignant melanoma within the small intestine [18].

Metastatic melanoma is one of the most common malignancies associated with spread to the GI tract. An autopsy series of 216 patients with advanced malignant melanoma at Roswell Park Memorial Institute suggested that GI metastasis is second only to the lung in sites of organ metastatic disease [6]. The most common sites of metastases were the lymph nodes $(73.6 \%)$ and lungs $(71.3 \%)$, followed by the liver $(58.3 \%)$, brain $(54.6 \%)$, bone $(48.6 \%)$, and adrenal glands $(46.8 \%)$. The incidence of GI metastases was $43.5 \%$ [6]. Multiple organ involvement of metastases was common $(95 \%)$. The distribution of GI organ metastases in this series was as follows: liver, 58.3\%; peritoneum, $42.6 \%$; pancreas, 37.5\%; small bowel, 35.6\%; spleen, 30.6\%; colon, $28.2 \%$; stomach, $22.7 \%$; oral cavity and esophagus, $9.3 \%$; and biliary tract, $8.8 \%$. A large review of autopsies from Memorial Sloan Kettering Cancer Center previously found the incidence of GI metastases to be as follows: liver, $68 \%$; small bowel, $58 \%$; colon, $22 \%$; stomach, $20 \%$; duodenum, $12 \%$; rectum, $5 \%$; esophagus, $4 \%$; and anus, $1 \%$ [7]. In ocular malignant melanoma, the most common site of metastasis was the liver, and single organ involvement was found in almost one third of patients. In a retrospective review of 230 patients with malignant melanoma, metastasis to the small bowel was found in $7.4 \%$ based on CT scanning [8]. Similarly, the 7-year experience at Tianjin Medical University Cancer Hospital (1989-1996) found small bowel metastases in $7.8 \%$ of malignant melanomas detected during that period. [9]

In contrast to secondary metastases, primary Small Bowel Melanoma is exceptional. The rarity of such tumors is not unexpected and can be explained by the lack of melanocytes in the small intestine, in contrast to the anorectum and even the esophagus where these cells are often naturally present [3].

It is very difficult to differentiate between primary and secondary Small Bowel Melanoma [3], [10]. The clinical importance of this distinction lies within the differential in prognosis. Prognosis is worse for primary intestinal melanomas which tend to grow faster and more aggressively than metastatic tumors perhaps due to the rich lymphovascular supply available in the intestinal mucosa [11]. In terms of prognosis, both primary and secondary GI malignant melanomas are worse than the conventional cutaneous equivalents, with a 5 -year survival of only $10 \%$ and median survival of 4-6 months [12].

To determine whether the small intestinal malignancy is a primary lesion Blecker et al. propose the following criteria for the diagnosis of primary melanoma of the small bowel: 1) presence of a solitary mucosal lesion in the intestinal epithelium, 2) absence of melanoma or atypical melanocytic lesions of the skin and 3) presence of intramucosal melanocytic lesions in the overlying or adjacent intestinal epithelium [13].

Several studies have reported about the efficacy and improvement in mortality of the surgical resection for melanoma metastases in both the gastrointestinal tract and other distant sites. Hao et al described 3 patients with melanoma metastatic to the small bowel, all of whom underwent surgical resection [9]. Two patients remained well 6 and 2 years after surgery, respectively, whereas 1 patient died of metastatic melanoma within the abdomen 4 years after surgery. Ollila et al reported on a retrospective review of 124 of 6509 melanoma patients who had GI tract metastases at the John Wayne Cancer Institute from 1971 through 1994. Of these 124 patients, 69 (56\%) underwent surgical exploration of the abdomen, of which 46 (67\%) had curative resection and $23(33 \%)$ had a palliative procedure only. Ollila et al. presented a study, where 67 (97\%) of 69 surgical patients experienced postoperative relief of their presenting GI tract symptoms [14]. Certain studies claim that gastrointestinal melanomas with unknown primaries may be metastatic, with either unidentified or spontaneously regressed cutaneous malignancy. In a study consisting of 437 cutaneous melanoma cases, $12.3 \%$ manifested at least partial regression [17].

In 2005, Krüger et al. reviewed 18 cases of primary malignant melanoma of the small bowel. In 5 of these cases a follow-up of 12 months or longer is reported; 2 of these had tumor recurrence [18]. Whether the often early recurrence reflects a poor prognosis of primary melanoma of the small intestine or a sign of other primary origin remains unclear. 


\section{International Journal of Science and Research (IJSR) \\ ISSN (Online): 2319-7064}

Index Copernicus Value (2013): 6.14 | Impact Factor (2015): 6.391

\section{Conclusions}

In this article we report the case of a cystic and mostly necrotic mass with histological findings and immune histochemical assays compatible with the melanoma involving the small bowel.

Given that most gastrointestinal melanomas are metastatic, to define the diagnosis of a primary intestinal melanoma accurate medical history investigation, physical examination and imaging studies are essential. In the setting of GIT melanomas with unknown primaries, the most accepted explanation is the model of tumor regression. In fact, some researchers consider all gastrointestinal tract melanomas to be metastatic in origin, based on the fact that some cutaneous melanomas suffer spontaneous regression. Nevertheless, the presence of a precursor lesion or melanosis further suggests the possibility that there might be primary gastrointestinal tract melanomas [17].

Although we consider our case to be primary disease, the possibility of metastasis from an otherwise regressed cutaneous melanoma cannot be completely excluded.

In conclusion, primary Small Bowel Melanoma is a rare entity, which can exist asymptomatically for long periods of time and is often diagnosed at an advanced stage, when there are limited treatment options.

\section{References}

[1] Reintgen DS, Thompson W, Garbutt J, et al. Radiologic, endoscopic, and surgical considerations of melanoma metastatic to the gastrointestinal tract. Surgery 1984;95:635-9. [PubMed]

[2] Chang AE, Karnell LH, Menck HR. The National Cancer Data Base report on cutaneous and noncutaneous melanoma: a summary of 84,836 cases from the past decade. The American College of Surgeons Commission on Cancer and the American Cancer Society. Cancer 1998;83:1664-78. [Crossref] [PubMed]

[3] Poggi SH, Madison JF, Hwu WJ, et al. Colonic melanoma, primary or regressed primary. J Clin Gastroenterol 2000;30:441-4. [Crossref] [PubMed]

[4] Schuchter LM, Green R, Fraker D. Primary and metastatic diseases in malignant melanoma of the gastrointestinal tract. Curr Opin Oncol. 2000;12: 181185.

[5] Folz BJ, Niemann AM, Lippert BM, Werner JA. Primary mucous membrane melanoma of the base of the tongue [in German]. Laryngorhinootologie. 1998;77:226-230

[6] Patel JK, Didolkar MS, Pickren JW, Moore RH. Metastatic pattern of malignant melanoma: a study of 216 autopsy cases. Am J Surg. 1978;135:807- 810.

[7] DasGupta TK, Brasfield RD. Metastatic melanoma of the gastrointestinal tract. Arch Surg. 1964;88:969-973.

[8] Kawashima A, Fishman EK, Kuhlman JE, Schuchter LM. CT of malignant melanoma: patterns of small bowel and mesenteric involvement. J Comput Assist Tomogr. 1991;15:570-574.
[9] Hao XS, Li Q, Chen H. Small bowel metastases of malignant melanoma: palliative effect of surgical resection. Jpn J Clin Oncol. 1999;29:442-444

[10] Amar A, Jougon J, Edouard A, et al. Primary malignant melanoma of the small intestine. Gastroenterol Clin Biol 1992;16:365-7. [PubMed]

[11]Liang KV, Sanderson SO, Nowakowski GS, et al. Metastatic malignant melanoma of the gastrointestinal tract. Mayo Clin Proc 2006;81:511-6. [Crossref] [PubMed]

[12] Schuchter LM, Green R, Fraker D. Primary and metastatic diseases in malignant melanoma of the gastrointestinal tract. Curr Opin Oncol 2000;12:181-5. [Crossref] [PubMed]

[13]Blecker D, Abraham S, Furth EE, Kochman ML. Melanoma in the gastrointestinal tract. Am J Gastroenterol. 1999;94:3427-3433.

[14] Ollila DW, Essner R, Wanek LA, Morton DL. Surgical resection for melanoma metastatic to the gastrointestinal tract. Arch Surg. 1996;131:975-980.

[15] Atmatzidis KS, Pavlidis TE, Papaziogas BT, Papaziogas TB. Primary malignant melanoma of the small intestine: report of a case. Surg Today. 2002;32:831833. [PubMed]

[16] Ihde JK, Coit DG. Melanoma metastatic to stomach, small bowel, or colon. Am J Surg. 1991;162:208211. [PubMed]

[17] McGovern VJ. Spontaneous regression of melanoma. Pathology. 1975;7:91-99. [PubMed]

[18] Krüger S, Noack F, Blöchle C, Feller AC: Primary malignant melanoma of the small bowel. A case report and review of the literature. Tumori 2005;91:73-76.

[19]Bloch T, Tejada E, Brodhecker C: Malignant melanoma in Meckel's diverticulum. Am $\mathrm{J}$ Clin Pathol 1986;86:231-234.

[20] Bender GN, Maglinte DD, McLarney JH, et al. Malignant melanoma: patterns of metastasis to the small bowel, reliability of imaging studies, and clinical relevance. Am J Gastroenterol 2001;96:2392-400.

\section{Author Profile}

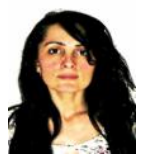

Alma Llukacaj received the Master Degree in Genera Surgery in February 2008. Now, she is in the final process of getting the $\mathrm{PhD}$ in General Surgery. From June 2001, she works as attendant general surgeon at the Hospital University Center "Mother Theresa" in Tirana, Albania. 